\title{
Conventional Thermal versus Water-Cooled Genicular Nerve Radiofrequency Lesioning: A Retrospective Chart Review
}

\author{
Cristina A. Shea, MD, Sara M. Burns, MS, Michael A. Pitts-Kiefer MD, and Shihab U. Ahmed, MD
}

Background: Genicular nerve radiofrequency lesioning (RFL) is an interventional approach to chronic knee pain. It is currently unknown whether conventional thermal RFL (CT-RFL) or watercooled RFL (WC-RFL) yields better outcomes.

Objective: The objective of this research was to analyze and compare outcomes of genicular nerve conventional thermal radiofrequency lesioning (CT-RFL) vs water-cooled radiofrequency lesioning (WC-RFL) for the treatment of chronic knee pain.

Study Design: We used retrospective chart review.

Setting: The research took place in an outpatient pain clinic at a large academic medical center.

Methods: Patients who participated in the study were those aged 18 and older who received genicular nerve RFL for chronic knee pain between January 1, 2014 and December 31, 2016. Random intercepts models were used to examine Visual Analog Scale (VAS) pain scores across the first year of follow-up, adjusting for age, gender, and prior history of knee surgery.

Results: Overall, VAS scores were significantly reduced from baseline (mean $=6.66$, standard deviation $[S D]=1.36$ ) by 1.46 points during the first follow-up month (95\% confidence interval [Cl], 0.6-2.3, $P=.001$ ), 2.22 points during the second follow-up month $(95 \% \mathrm{Cl}, 1.4-3.1, P=<.001)$, and 1.24 points during the sixth follow-up month (95\% $\mathrm{Cl}, 0.1-2.4, P=.035)$ but were not significantly reduced at other months during the one-year followup time period. There was no statistically significant difference in follow-up pain scores (mean difference $=0.73,95 \% \mathrm{Cl},-0.14-1.59, P=.116)$ or rates of complications $(P=.10,2$-tailed Fisher exact test) between RFL types.

Limitations: Study shortcomings include patient loss to follow-up, heterogeneity of CT-RFL techniques, and heterogeneity of study patients.

Conclusions: Genicular RFL is a promising strategy for long-term management of treatment-resistant chronic knee pain. In this study, no significant difference in outcomes was detected between CT-RFL and WC-RFL techniques. Larger prospective studies are warranted to compare outcomes of these techniques and guide future care.

Key words: Radiofrequency lesioning, knee pain, chronic pain, osteoarthritis, genicular, cooled radiofrequency lesioning, water-cooled radiofrequency lesioning, conventional radiofrequency lesioning
From : Center for Pain Management, Department of Anesthesia, Critical Care, and Pain Medicine, Massachusetts General Hospital and Harvard Medical School, Boston, MA

Author for correspondence: Cristina A. Shea, MD

Address: Center for Pain Medicine, Massachusetts General Hospital and Harvard Medical School, 15 Parkman St., Boston, MA 02114 E-mail: cshea17@partners.org

Disclaimer: There was no external funding in the preparation of this manuscript. Conflict of interest: Each author certifies that he or she, or a member of his or her immediate family, has no commercial association (i.e., consultancies, stock ownership, equity interest, patent/licensing arrangements, etc.) that might pose a conflict of interest in connection with the submitted manuscript.
Nearly $10 \%$ of older adults have experienced knee pain on most days for at least a month, according to a US population-based cohort study (1). The most common cause of chronic knee pain in adults age 65 years and older is osteoarthritis (OA), which affects $10 \%$ to $15 \%$ of individuals (2). This prevalence is expected to increase as the population of older adults grows and rates of obesity, a major risk factor for $\mathrm{OA}$, continue to rise $(3,4)$. At least half of patients with $\mathrm{OA}$ of the knee report restriction of daily activity secondary to 
pain (5). Therefore, effective pain management is key to maintaining independence and maximizing quality of life in this patient population.

Conservative treatment for chronic knee pain includes physical therapy and oral pain medications such acetaminophen or nonsteroidal antiinflammatory drugs (NSAIDs) (6). If these fail to yield sufficient pain relief, procedures such as acupuncture or intraarticular steroid or hyaluronic acid injections are sometimes employed (7). Ultimately, many with intractable pain secondary to OA undergo total knee replacement. While total knee replacement has been shown to yield greater pain relief than conservative nonsurgical treatments, it carries the operative risk of anesthesia and postsurgical risks such as deep venous thrombosis and joint infection (8), and therefore is not an option for some medically complex patients. Additionally, it is estimated that between $7 \%$ and $20 \%$ of patients have persistent pain despite undergoing total knee replacement (9).

Genicular nerve radiofrequency lesioning (RFL) may provide long-term relief for patients with chronic knee pain who have failed to find sufficient relief from other treatment modalities. In this outpatient procedure that requires only local anesthetic, electrodes that release radiofrequency ( $R F)$ waves are used to thermally ablate the sensory nerves carrying pain sensation from the knee joint. While a number of case series and randomized controlled trials have reported sustained improvement in pain scores following genicular nerve $\mathrm{RFL}$, there is significant heterogeneity in techniques among these studies $(10,11)$. One unanswered question is whether conventional thermal RFL (CTRFL) or water-cooled RFL (WC-RFL) leads to better outcomes. In contrast to CT-RFL, in WC-RFL the electrode is actively cooled, allowing for a longer duration flow of RF current and resulting in larger radius lesions. A recent anatomic study by Franco et al (12) proposes that CT-RFL lesions may be too small to effectively ablate genicular nerves. WCRFL may result in pain relief of greater magnitude and longer duration, but is significantly more costly. Making an evidence-based decision between these 2 techniques is currently difficult, as no comparison of their outcomes has been published to date. This retrospective chart review aimed to address this knowledge gap by examining outcomes of patients treated in an outpatient pain clinic with either CT-RFL or WC-RFL for chronic knee pain.

\section{METHODS}

\section{Study Patients}

Medical records of all patients aged 18 and older who received genicular nerve RFL for chronic knee pain at the Massachusetts General Hospital Pain Clinic between January 1, 2014 and December 31, 2016 were reviewed for this study. The study was approved by the Massachusetts General Hospital Institutional Review Board (IRB).

\section{Diagnostic Genicular Nerve Block \& RFL Tech- niques}

All patients included in this study underwent diagnostic genicular nerve block with $\geq 50 \%$ relief, followed by either CT-RFL or WC-RFL at the following visit. All procedures were fluoroscopy-guided. For both nerve blocks and RFL procedures, the superior medial (SM) and superior lateral (SL) genicular nerves were targeted just superiorly to the medial and lateral epicondyles of the femur, and the inferior medial (IM) genicular nerve was targeted at the medial condyleshaft intersection of the tibia. Anesthetic type and volume used for diagnostic blocks and to anesthetize these 3 nerve sites prior to RFL denervation varied based on provider preference. For CT-RFL procedures, denervation was performed at either 80 or 85 degrees Celsius for either 60 or 90 seconds. For WC-RFL procedures, denervation was performed at 60 degrees for 150 seconds (see Table 1 for details).

\section{STUDY DESIGN}

The principal aim of this retrospective chart review was to compare outcomes between patients who underwent CT-RFL and WC-RFL. The primary outcome of interest was improvement in Visual Analog Scale (VAS) pain score from baseline to follow-up visits within the year following the RFL procedure. For each patient, the VAS score recorded the day of the RFL prior to the procedure was recorded as their baseline VAS score. If this score was not available, the VAS score at the previous clinic visit was used as the baseline. Reported VAS scores from clinic notes during the year following the RFL were then recorded as follow-up pain scores. If a VAS score was reported 
as a range, the midpoint was used (e.g., 3.5 for a reported VAS score of 3-4). Additionally, if a patient reported multiple VAS scores in a given follow-up month, the scores were averaged to yield one pain score for the month. Data was also collected from the electronic medical record on postprocedure complications and baseline patient characteristics, including diagnosis, patient age, gender, and prior knee surgery on the knee receiving the RFL.

\section{Statistical Analysis}

No a priori statistical power calculation was performed given the retrospective nature of this study. The analysis was conducted on all available data. All analyses were conducted using $R$ statistical software (RStudio, version 3.2.2; R Foundation for Statistical Computing, Vienna, Austria).

Means (standard deviation [SD]) and frequency counts (\%) of baseline patient characteristics were determined, then stratified by RFL type. Comparisons of frequency counts between RFL types were made using the Fisher exact test. Random intercepts models with patient as a random effect were constructed to examine VAS scores over time. The models apply baseline pain score as a reference for each patient and are adjusted for age, gender, and prior knee surgery on the procedure knee. The models allow for inclusion of VAS scores from multiple procedures for individuals who underwent multiple RFLs. All hypothesis testing was 2-tailed with significance interpreted as $P<.05$.

\section{RESULTS}

A total of 47 patients received genicular nerve RFL procedures during the study period. Of these patients, 38 underwent a single RFL and 9 underwent multiple RFL procedures (5 underwent 2 RFL procedures, 2 underwent 4 RFL procedures, and 2 underwent 5 RFL procedures). Table 2 shows baseline characteristics of study participants (using data from the first procedure for individuals who underwent multiple RFL procedures). Patients in the WC-RFL group were slightly older than those in the CT-RFL group (66.7 vs 60.7 years, standardized mean difference $[S M D]=0.388$ ) and a smaller proportion was female $(21.7 \%$ vs. $45.8 \%$, SMD $=0.527)$. The majority of patients in both groups
Table 1. Details of RFL procedures by RFL type.

\begin{tabular}{|l|c|c|}
\hline & CT-RFL $(\mathbf{n}=\mathbf{3 5})$ & WC-RFL $(\mathbf{n}=\mathbf{3 1})$ \\
\hline & $16(\mathrm{n}=7)$ & \\
$18(\mathrm{n}=3)$ & $20(\mathrm{n}=21)$ \\
$22(\mathrm{n}=3)$ & $17(\mathrm{n}=31)$ \\
Cannula Size (gauge) & Not recorded (n=1) & \\
\hline Active Tip Size (mm) & $\begin{array}{c}10(\mathrm{n}=28) \\
5(\mathrm{n}=2) \\
\text { Not recorded (n=5) }\end{array}$ & $4(\mathrm{n}=31)$ \\
\hline $\begin{array}{l}\text { Denervation } \\
\text { Temperature }\left({ }^{\circ} \mathrm{C}\right)\end{array}$ & $\begin{array}{c}80(\mathrm{n}=34) \\
85=1)\end{array}$ & $60(\mathrm{n}=31)$ \\
\hline $\begin{array}{l}\text { Duration of } \\
\text { Denervation (s) }\end{array}$ & $\begin{array}{c}60(\mathrm{n}=26) \\
90(\mathrm{n}=9)\end{array}$ & $150(\mathrm{n}=31)$ \\
\hline
\end{tabular}

Abbreviations: CT-RFL, conventional thermal radiofrequency lesioning; WCRFL, water-cooled radiofrequency lesioning.

Table 2. Baseline characteristics of study participants.

\begin{tabular}{|l|c|c|c|c|}
\hline & $\begin{array}{c}\text { Overall } \\
(\mathbf{n}=47)\end{array}$ & $\begin{array}{c}\text { CT-RFL } \\
(\mathbf{n}=\mathbf{2 4})\end{array}$ & $\begin{array}{c}\text { WC-RFL } \\
(\mathbf{n}=\mathbf{2 3})\end{array}$ & SMD \\
\hline Age (mean [SD]) & $63.6(15.5)$ & $60.7(15.5)$ & $66.7(15.2)$ & 0.388 \\
\hline $\begin{array}{l}\text { Female Gender (n } \\
[\%])\end{array}$ & $16(34.0)$ & $11(45.8)$ & $5(21.7)$ & 0.527 \\
\hline $\begin{array}{l}\text { Diagnosis of } \\
\text { Osteoarthritis (n [\%]) }\end{array}$ & $32(68.1)$ & $14(58.3)$ & $18(78.2)$ & 0.333 \\
\hline $\begin{array}{l}\text { Prior Knee Surgery } \\
\text { on RFL Knee (n [\%]) }\end{array}$ & $20(42.6)$ & $8(33.3)$ & $12(52.2)$ & 0.388 \\
\hline
\end{tabular}

Abbreviations: CT-RFL, conventional thermal radiofrequency lesioning; RFL, radiofrequency lesioning; SMD, standardized mean difference; SD, standard deviation; WC-RFL, water-cooled radiofrequency lesioning.

underwent RFL for pain secondary to OA (32 of 47 patients, $58.3 \%$ in the CT-RFL group and $78.2 \%$ in the WC-RFL group, SMD = 0.333). Other diagnoses were post-total knee replacement pain $(n=13,5$ in the CT-RFL group and 8 in the WC-RFL group) and chronic neuropathic pain thought to be related to prior interventions $(n=2$, one in each of the CT-RFL and WC-RFL groups). There was a greater proportion of patients in the WC-RFL group with a history of knee surgery on the procedure knee $(52.2 \%$ vs $33.3 \%$ in the CT-RFL group, SMD $=0.388$ ).

Data from all procedures with available follow-up VAS scores was used in the models. There were no follow-up pain scores for 10 out of 66 procedures due to loss to follow-up. Mean raw VAS scores among all patients are depicted in Fig. 1. Adjusted VAS scores were significantly reduced from baseline (mean $=6.66$, 
$\mathrm{SD}=1.36)$ by 1.46 points during the first follow-up month $(95 \%$ confidence interval $[\mathrm{Cl}], 0.6-2.3, P=$ $.001), 2.22$ points during the second follow-up month $(95 \% \mathrm{Cl}, 1.4-3.1, P=<.001)$, and 1.24 points during the sixth follow-up month $(95 \% \mathrm{Cl}, 0.1-2.4, P=.035)$ but were not significantly reduced at other months during the one-year follow-up time period.

Mean raw VAS scores stratified by RFL type are depicted in Fig. 2. Patients treated with CT-RFL appeared to have slightly lower adjusted VAS scores over time (greater pain relief) than patients treated with the WC-RFL; however, the difference was not statistically significant (mean difference $=0.73$, $95 \% \mathrm{Cl},-0.14-1.59, P=.116)$. Four postprocedure complications were reported in the CT-RFL group (3 cases of postprocedure neuritis, 1 case of a knee effusion) vs none in the WC-RFL group, though this difference was not statistically significant $(P=.10$, 2-tailed Fisher exact test).

\section{DISCUSSION}

In this retrospective chart review, genicular nerve RFL resulted in significantly improved pain scores during the first, second, and sixth follow-up months. The magnitude of pain reduction was $>1$ VAS point during all 3 months, which has previously been determined as the minimally clinically important difference in patients with chronic musculoskeletal pain (osteoarthritis, rheumatoid arthritis, and anky-

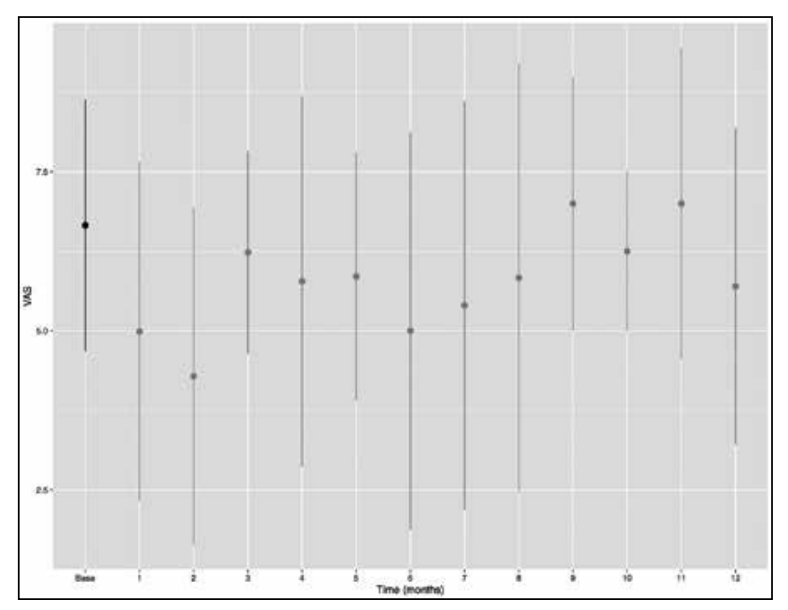

Fig. 1. Raw VAS scores of all RFL procedures. losing spondylitis) (13). Pain reduction was $>2$ VAS points during the second follow-up month only, which Dworkin et al argue corresponds to a more clinically significant pain rating of "much improved" (14). While Dworkin et al argue that one point may be a less important change, in this setting a pain reduction of one VAS point is likely still clinically meaningful given that a single RFL procedure can be used as an add-on therapy to other pain-relief techniques (medication, physical therapy, etc.).

Alternatively, VAS scores were not significantly decreased from baseline between the third and fifth months or after 6 months. As shown in Fig. 3, excluding the third follow-up month, the number of patients who returned for follow-up during these months was less than the number who returned during the first, second, and sixth follow-up months. It is difficult to know whether the smaller number of patients who returned for follow-up during these months were representative of the entire cohort. It is possible that those who returned to the clinic more regularly had poorer response to the RFL and therefore returned more often to seek alternate treatment options. This potential sample bias could explain the loss of improvement in pain scores observed between the third and fifth months, but resurgence in significant improvement observed during the sixth month, when more patients returned to the clinic for routine follow-up.

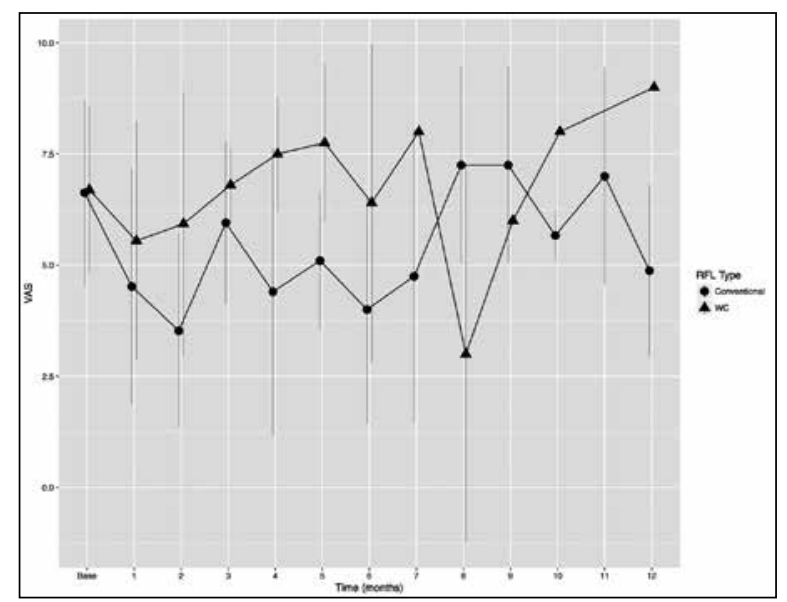

Fig. 2. Raw VAS scores by RFL type. 
As a result of small sample size after the sixth month, it is hard to draw conclusions about the true duration of RFL-induced pain relief in this cohort. The significant improvement from baseline observed at the sixth month, though, may suggest a duration of efficacy of at least that length. Few studies have examined outcomes of genicular nerve RFL procedures past 3 to 6 months. Pineda et al found in their prospective study of 25 patients with OA treated with CT-RFL that $88 \%$ reported at least $50 \%$ improvement in pain at one month. This decreased to $64 \%$ at 6 months and $32 \%$ at 12 months (15). Though they used a different RFL technique (pulsed RFL, with 20 milliseconds of $120045-\mathrm{V}$ pulses from a $10-\mathrm{mm}$ active tip), Masala et al found that at the one year follow-up, the mean VAS score was still significantly improved from baseline $(2.3 \pm 0.6$ from $6.8 \pm 0.8)$ in their prospective case series of 40 patients with pain secondary to $\mathrm{OA}(16)$. These prospective studies suggest that RFL techniques could result in analgesia for at least 6 months to 1 year, agreeing with the findings of this retrospective study. Given the difference in techniques, though, these studies raise the question of whether duration of pain relief may differ by RFL type, a principal question driving the present study.

Gupta et al recently reviewed the available literature on conventional, pulse, and cooled RFL for chronic knee pain. While the majority of studies reported positive outcomes, there was significant heterogeneity in methodology, and no studies were found that attempted a head-to-head comparison of different RFL techniques (11). This retrospective study is the first study directly comparing outcomes between 2 RFL methods. No difference was detected between follow-up VAS scores in patients who were treated with CT-RFL vs WC-RFL, though it appeared that the patients treated with CT-RFL had slightly lower VAS scores over time (Fig. 2). Additionally, while there were more postprocedure complications among the CT-RFL group (4 vs. 0 in the WC-RFL group), this different was not significant. Thus, no overall differences between these 2 techniques were detected. This study sets the groundwork for future comparative studies, which are vital for forming evidence-based treatment guidelines and delivering cost-effective care.

As previously mentioned, the main shortcoming of this study is limited follow-up data. Patients included

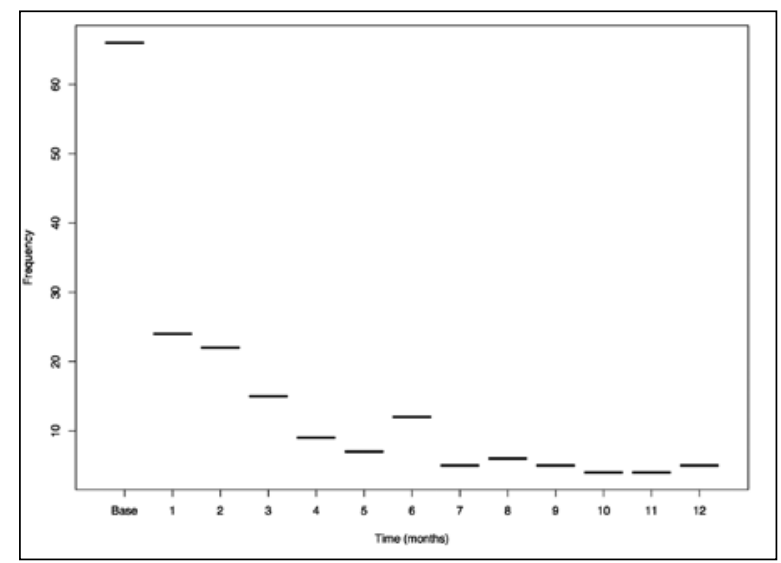

Fig. 3. Number of VAS scores available for analysis, by follow-up month.

in this study were not required to have a predetermined frequency of follow-up visits. All patients with at least one follow-up VAS score were included in order to maximize sample size. The small sample size at each follow-up month resulted in sizeable error of adjusted pain scores. Therefore, it is hard to know whether there was truly no difference in followup pain scores between the 2 RFL types or whether there simply was not sufficient power to detect a difference. A related issue previously alluded to is that it is likely that patients who returned for follow-up less frequently had different outcomes than those who regularly returned. Those who returned to the clinic less frequently to report VAS scores may have had less pain and felt that returning to the doctor was unnecessary, or alternatively may have been dissatisfied with the outcome of the procedure and decided to pursue care elsewhere. A next step to build upon the findings of this study would be to conduct a prospective study with frequent predetermined follow-up times for all patients.

Another study limitation is that while the technique for WC-RFL procedures was standardized, methods used to carry out CT-RFL procedures in this patient cohort varied between practitioners, as shown in Table 1. In addition to procedural heterogeneity, there was also heterogeneity in the patient population. While most patients in both RFL groups had the diagnosis of $O A$, patients with other diagnoses (post-total knee replacement pain and chronic neuropathic pain) 
were included in the study to maximize sample size. The frequency of these diagnoses varied somewhat between RFL treatment groups. In addition, data was not collected on other comorbid medical conditions or pain conditions. Since it is possible that outcomes may vary significantly based on the underlying pain generator, future studies should examine patients with these diagnoses separately. In particular, one might speculate that RFL may be less effective among patients with multifocal pain secondary to centrally driven pain syndromes than among patients with localized pain only in the knee. Given the small sample size of this study, there was not sufficient power to perform a subgroup analysis of this type. A larger sample size would allow subgroup analysis in order to see if certain subgroups, such as those without comorbid pain conditions, are more likely to benefit from this therapy. Finally, due to methodological difficulty, this study did not consider the potential confounder of other treatment modalities during the study period. Since many patients also sought care

\section{REFERENCES}

1. Felson D, Naimark A, Anderson J, Kazis L, Castelli W, Meenan RF. The prevalence of knee osteoarthritis in the elderly: The Framingham Osteoarthritis Study. Arthritis Rheum 1987; 30:914918.

2. Garstang SV, Stitik TP. Osteoarthritis: Epidemiology, risk factors, and pathophysiology. Am J Phys Med Rehabil 2006; 85:S2S11.

3. Zhang Y, Jordan JM. Epidemiology of osteoarthritis. Clin Geriatr Med 2010; 26:355-369.

4. Felson D. Epidemiology of hip and knee osteoarthritis. Epidemiol Rev 1988; 10:1-28. doi:10.1093/oxfordjournals.epirev.a036019.

5. Peat G, McCarney R, Croft P. Knee pain and osteoarthritis in older adults: A review of community burden and current use of primary health care. Ann Rheum Dis 2001; 60:91-97.

6. American Academy of Orthopaedic Surgeons. Treatment of Osteoarthritis of the Knee. Evidence-Based Guideline. $2^{\text {nd }}$ ed. www. aaos.org/cc_files/aaosorg/research/guidelines/treatmentofosteoarthritisofthekneeguideline.pdf. Date Published 05/18/2013. Date Accessed 06/02/2017.

7. Hochberg MC, Altman RD, April KT, et al. American College of Rheumatology 2012 recommendations for the use of nonpharmacologic and pharmacologic therapies in osteoarthritis of the hand, hip, and knee. Arthritis Care Res (Hoboken) 2012; 64:465474. http://www.ncbi.nlm.nih.gov/pubmed/22563589. Date Accessed 06/02/2017.

8. Skou ST, Roos EM, Laursen MB, et al. A randomized, controlled trial of total knee replacement. N Engl J Med 2015; 373:15971606.

9. Wylde V, Hewlett S, Learmonth ID, Dieppe P. Persistent pain after joint replacement: Prevalence, sensory qualities, and post- outside the studied hospital network, it would have been difficult to fully assess other therapies during the study period retrospectively via the available electronic medical record.

Despite these limitations, this study is notable for being the first study to directly compare 2 modalities of genicular RFL. Another key strength is the oneyear follow-up duration, which is longer than most previously published studies examining the efficacy of genicular RFL (11). In this study, genicular nerve RFL resulted in significantly improved pain scores during the first, second, and sixth follow-up months. These findings suggest that genicular nerve RFL can bring clinically meaningful, long-term relief to patients with chronic knee pain who have failed to find sufficient relief with other treatment modalities. Future prospective studies with more standardized RFL procedures and more homogeneous study populations are warranted to build on these findings and guide future clinical care. operative determinants. Pain 2011; 152:566-572.

10. Bhatia A, Peng P, Cohen SP. Radiofrequency procedures to relieve chronic knee pain: An evidence-based narrative review. Reg Anesth Pain Med 2016; 41:501-510.

11. Gupta A, Huettner DP, Dukewich M. Comparative effectiveness review of cooled versus pulsed radiofrequency ablation for the treatment of knee osteoarthritis: A systematic review. Pain Physician 2017; 20:155-171. http://www.ncbi.nlm.nih.gov/ pubmed/28339430. Date Accessed 06/02/2017.

12. Franco CD, Buvanendran A, Petersohn JD, Menzies RD, Menzies LP. Innervation of the anterior capsule of the human knee: Implications for radiofrequency ablation. Reg Anesth Pain Med 2015; 40:363-368.

13. Salaffi F, Stancati A, Silvestri CA, Ciapetti A, Grassi W. Minimal clinically important changes in chronic musculoskeletal pain intensity measured on a numerical rating scale. Eur J Pain 2004; 8:283-291.

14. Dworkin RH, Turk DC, Wyrwich KW, et al. Interpreting the clinical importance of treatment outcomes in chronic pain clinical trials: IMMPACT recommendations. J Pain 2008; 9:105-121.

15. Santana Pineda MM, Vanlinthout LE, Moreno Martín A, van Zundert J, Rodriguez Huertas F, Novalbos Ruiz JP. Analgesic effect and functional improvement caused by radiofrequency treatment of genicular nerves in patients with advanced osteoarthritis of the knee until 1 year following treatment. Reg Anesth Pain Med 2017; 42:62-68.

16. Masala S, Fiori R, Raguso M, Morini M, Calabria E, Simonetti G. Pulse-dose radiofrequency for knee osteoartrithis. Cardiovasc Intervent Radiol 2014; 37:482-487. 